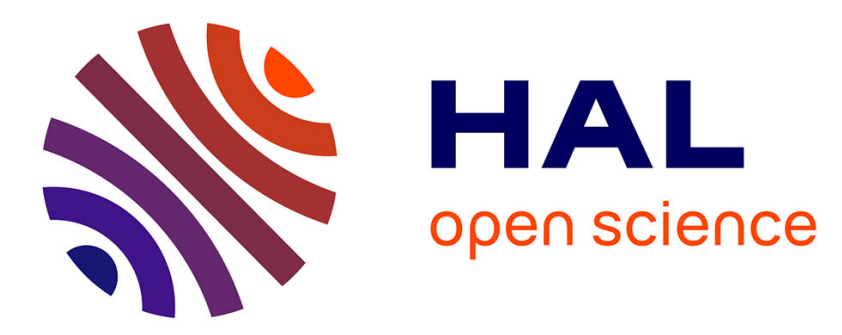

\title{
Specifications and Development of Interoperability Solution dedicated to Multiple Expertise Collaboration in a Design Framework
}

\author{
Alain Etienne, Emmanuel Guyot, Dimitri van Wijk, Lionel Roucoules
}

\section{- To cite this version:}

Alain Etienne, Emmanuel Guyot, Dimitri van Wijk, Lionel Roucoules. Specifications and Development of Interoperability Solution dedicated to Multiple Expertise Collaboration in a Design Framework. International Journal of Product Lifecycle Management, 2011, 5 (2/3/4), p. 272-274. hal-00749865

\section{HAL Id: hal-00749865 https://hal.science/hal-00749865}

Submitted on 8 Nov 2012

HAL is a multi-disciplinary open access archive for the deposit and dissemination of scientific research documents, whether they are published or not. The documents may come from teaching and research institutions in France or abroad, or from public or private research centers.
L'archive ouverte pluridisciplinaire HAL, est destinée au dépôt et à la diffusion de documents scientifiques de niveau recherche, publiés ou non, émanant des établissements d'enseignement et de recherche français ou étrangers, des laboratoires publics ou privés. 


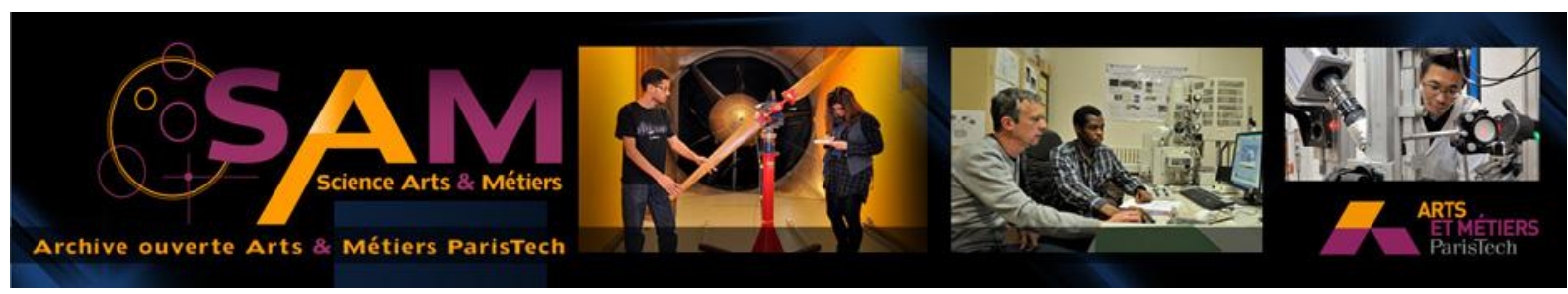

\section{Science Arts \& Métiers (SAM)}

is an open access repository that collects the work of Arts et Métiers ParisTech researchers and makes it freely available over the web where possible.

This is an author-deposited version published in: http://sam.ensam.eu Handle ID: . http://hdl.handle.net/10985/6296

\section{To cite this version :}

A. Etienne, E. Guyot, D. Van Wijk, et L. Roucoules, « Specifications and Development of Interoperability Solution dedicated to Multiple Expertise Collaboration in a Design Framework », 2011. 


\title{
Specifications and Development of Interoperability Solution dedicated to Multiple Expertise Collaboration in a Design Framework
}

\author{
A. Etienne, E. Guyot, D. Van Wijk and L. Roucoules
}

\begin{abstract}
This paper describes the specifications of an interoperability platform based on the PPO (Product Process Organization) model developed by the French community IPPOP in the context of collaborative and innovative design. By using PPO model as a reference, this work aims to connect together heterogonous tools used by experts easing data and information exchanges. After underlining the growing needs of collaborative design process, this paper focuses on interoperability concept by describing current solutions and their limits. Then a solution based on the flexibility of the PPO model adapted to the philosophy of interoperability is proposed. To illustrate these concepts, several examples are more particularly described (robustness analysis, CAD and Product Lifecycle Management systems connections).
\end{abstract}

Index Terms - Collaborative Design, Design Automation, Knowledge Modeling, Interoperability

\section{INTRODUCTION}

$\mathrm{I}_{\mathrm{c}}^{\mathrm{n}}$ $\mathrm{n}$ the framework of collaborative product design, which eases the emergence of innovative solutions and reduces product time cycle developments and its cost [1], sharing data and information between all experts and theirs dedicated tools is the main difficulty to overcome. Indeed, in this context of more complex and more multidisciplinary design, this collaboration gathers numerous experts handling heterogeneous views of the product and its manufacturing process. Moreover, the work of each expert is supported by several dedicated tools which have to be included into this framework too.

Three major solutions are commonly used to perform this data and information sharing:

(1) The first (depicted in Figure 1 in doted lines), the natural one, is based on direct exchanges between experts (during meetings, by mail, by phone, technical reports...) and between tools (by using formatted standard files as STEP for instance).

(2) The second consists in managing files transfers with PLM systems. In this solution, information which is hidden into its storing file is difficult to identify (semantically and technically) and to find. This difficulty is up with not open formats.

(3) The last one is based on information exchanges supported by a common data kernel. In this case, since this kernel only manages data, the functionalities of PLM systems are considered as one expert tool such as Computer Aided Design.

This work was partially supported by the Carnot TIE Program (a French multi expertise domains program regarding Technology and Innovation in Engineering).

A. Etienne is with Arts et Métiers ParisTech - LCFC Laboratory, 4 rue Augustin Fresnel, 57070 METZ TECHNOPOLE, France. Corresponding Author: phone: +33(0)3.87.37.54.88; e-mail: alain.etienne@ensam.eu).

E. Guyot and D. Wijk are with the University of Technology of Troyes - ICD/LASMIS Laboratory, 12 rue Marie Curie, BP 2060, 10010 TROYES, France.

L. Roucoules is with Arts et Métiers ParisTech - LSIS Laboratory, 2, cours des Arts et Métiers, 13617 AIX-EN-PROVENCE, France. 


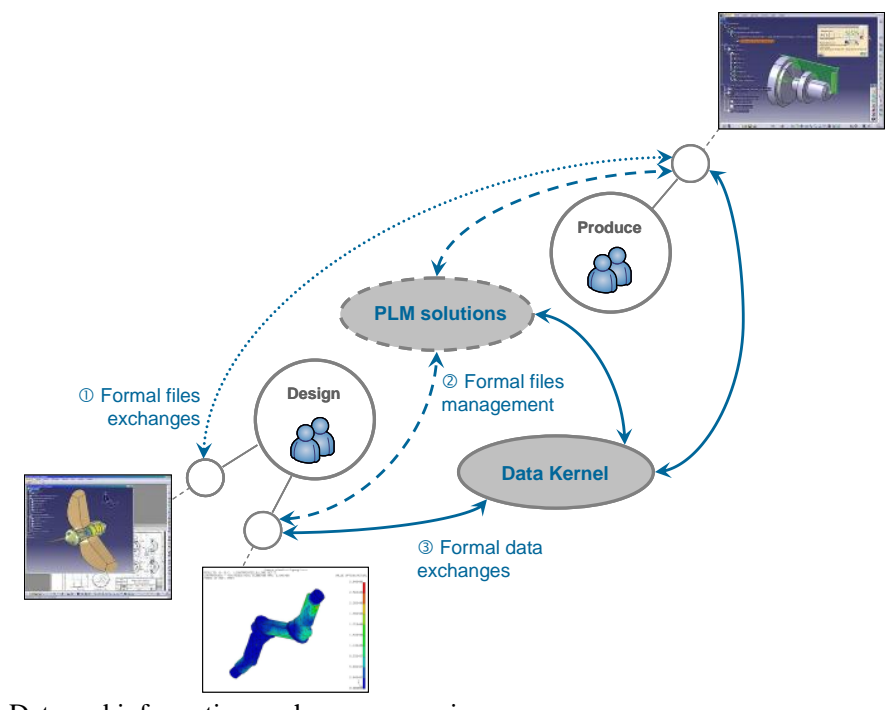

Figure 1: Collaborative Product Design: Data and information exchanges scenarios

Considering this context of collaborative design, the aim of this paper is to present specifications of a solution able to manage common data from several dedicated tools and how these heterogonous tools can access it considering interoperability constraints. Three applications (robustness analysis tool, CAD and PDM systems) are proposed to illustrate concepts and technical solutions of our proposed solution.

\section{PPO FRAMEWORK: THE FRENCH IPPOP PROJECT}

As explained in the previous section, PLM (and so PDM) systems, which only manage containers (i.e. shared files), seems to be limited in a collaborative design process where contents (and so, information and data) are more important [2]. In order to develop a framework allowing collaboration of multiple domains expertise in an innovative design, several French experts gather their needs, specifications and knowledge in the French national IPPOP project (Integration of Product Process and Organization for Performance enhancement in engineering).

Results of this project are currently available in the IPPOP Community Website [3] as an open source framework. This framework develops the resulting Product Process and Organization model, a kernel, allowing the integration of product and process knowledge into a manageable environment for concurrent engineering.

Since the aim of this paper is not the detailed explanation of the whole PPO model, the next section is more particularly focused on the product definition, underlining its flexibility and its relevance in a collaborative design context. Some examples of expert tools based on this framework are given too.

\section{A. Product modeling concepts:}

Since this model is dedicated to collaboration in innovative design, it has to meet two main requirements [4].

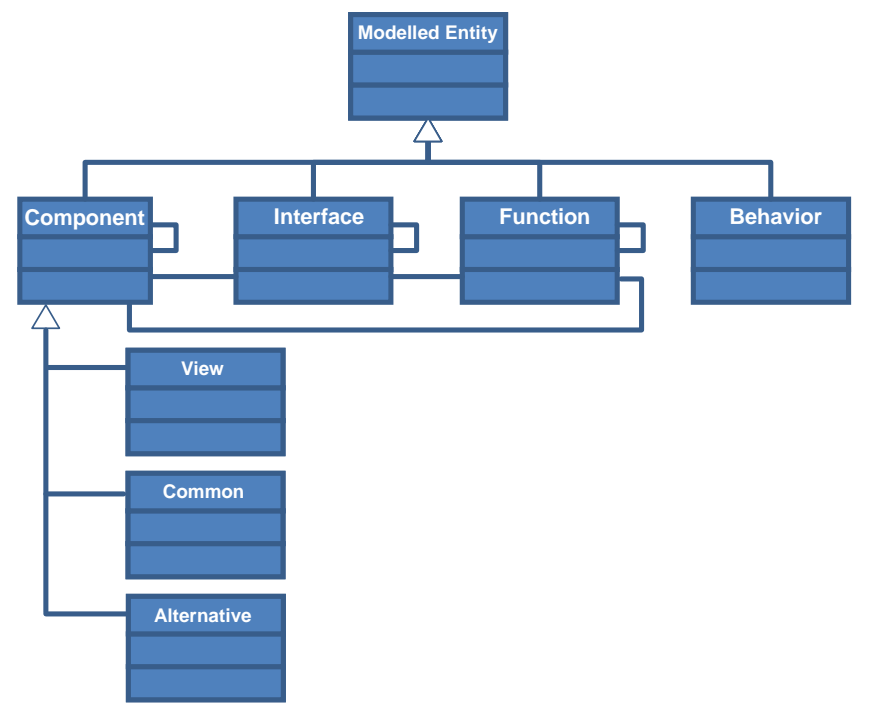

Figure 2: UML representation of the set of concepts composing the product model. Only the Component Object Class is detailed 
The first is to provide a model generic enough to be handled by any collaborating technical expertise. The second requirement is to develop a simple enough model to be handled in the earliest design steps, where the main product structures, functions and behaviors are not well established.

In order to be compliant with the know-how of all experts participating in the product life cycle, the design of this product model (partially illustrated in Figure 2) is voluntary reduced to four concepts: Component, Interface, Function and Behavior:

- The component object class models a partition of the product. Due to the recursive loop, the product structure can be divided into component which can be subdivided into subcomponents too. Moreover, since this concept can be particularized as a view, a common element or an alternative one, this model allows experts to share their specific point of view regarding a component. For instance, a component can concern the stress resistance and another one its manufacturability.

- The interface concept is a handler of a component, a property by which a component can be linked to one another. These interfaces can be, for example, mechanical (cinematic) or electrical handlers.

- The third concept, the function, is a relation linking at least two components through their interfaces. This concept quantifies this relation by formalizing the objective it has to reach.

- The last concept, the behavior, models a modal state inside the product lifecycle.

Shortly, these four concepts are enough to define the main product characteristics. Since this model can handle multiple expert views (thanks to the decomposition mechanism) and since its concepts are generic enough to be understood by any designers, this product model (and more globally the PPO model) can become the collaboration center between any stages of product design. The next section underlines this fact by enumerating some applications based on the PPO core (A more detailed example of how product is modeled in PPO is given in [4]).

\section{B. Examples of expert tools based on PPO model:}

Among the numerous applications developed in the IPPOP Project to show the connectivity and the genericity of its PPO model [5], three of them can be detailed:

- An application dedicated to the tolerance analysis, by integrating the results of MECAmaster which is a commercial tool developed to analyse the tolerance from the product structural description furnished by CATIA geometric modeller, in the PPO platform [6]. According to these references, the PPO platform (contrary to the most commercial solutions) can help designers to define geometric specifications on influent geometric elements and can support all concepts handled by tolerancing experts.

- A project planning application linked to the open source project management "planner" was developed too. With this solution, it is possible to view on this dedicated tool all projects, tasks and resources modeled and stored in the design process model of the PPO core.

- In this collaborative design framework, some conflicts can appear. In order to solve these troubles, a conflict management tool, called $\mathrm{CO}^{2} \mathrm{MED}$ was developed [7]. Based on the design process model and the organization model of the PPO core, this web site manage transactions between all actors involved in the design conflict to find a solution and to capitalize it.

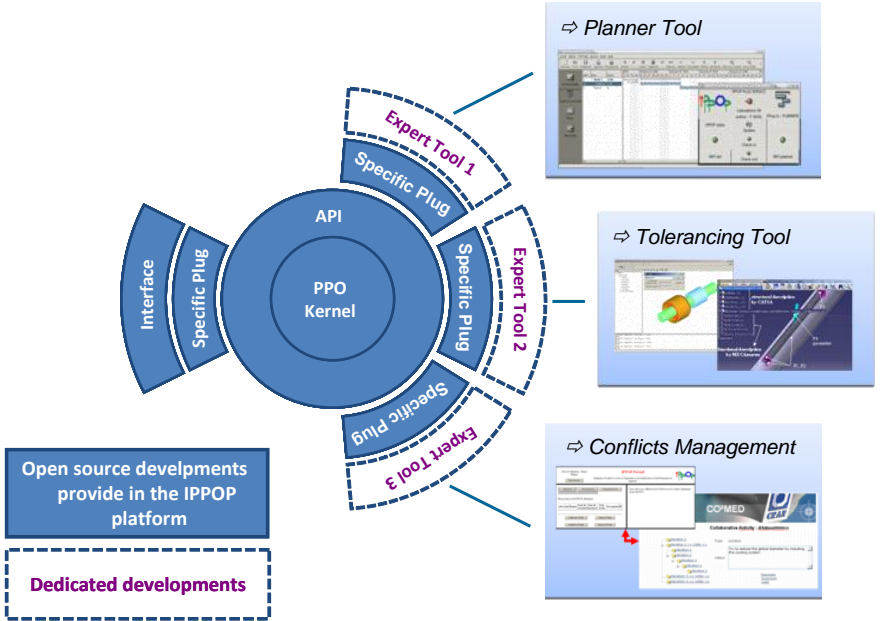

Figure 3: IPPOP Platform structure and illustrative expert tools based on the PPO kernel

The Figure 3 illustrates the structure of the IPPOP platform and how these expert tools are plugged to the PPO model. Thanks to dedicated plugs invocating several management functions (especially request functions gathered into one PPO API [3]) 
computer services can use concepts and data contained into an instantiation of the PPO model. That is the reason why this model is currently used by several expert tools, from the planning management to the tolerance analysis.

However these solutions, which incorporate the PPO core into their software developments, are still isolated. Although they handle the same concepts and data structure, they cannot really interoperate together. That is the reason why the aim of this work is to provide, in the same way of the IPPOP Project, a software infrastructure based on PPO kernel allowing interoperability between expert tools.

As a consequence, the further section deals with interoperability issues by focusing on: the conceptual view of what does interoperate mean, the different levels composing this concept, solutions available and their limits.

\section{INTEROPERABILITY: CONCEPTS, SOLUTIONS AND LIMITS}

Among the numerous definitions of the interoperability concept available in literature ([8][9][10][11][12] and [13] from the European Network of Excellence INTEROP), this paper considers the interoperability as "the ability of two or several systems or components to exchange information then to exploit information that has been exchanged"[9].

Moreover, according to several European works and frameworks (ATHENA [14], AIF [15] and EIF [16]), the interoperability concept can be decomposed in three different layers: organizational (the ability to understand the process), semantic (the ability to understand the idea/concept) and technical interoperability (the ability to understand the language and its syntax/grammar).

In the context of collaborative product design, although each stage has to be managed, only the technical view is currently regarded. The following sections of this paper deal with these layers by more particularly focusing on both semantic and technical stages.

\section{A. Semantic interoperability}

The semantic interoperability aims to "assure that exchanged information (regarding data, processes and applications) as the same meaning considering the point of view of both the sender and the receiver" [17]. This issue which is usually neglected in current works on collaborative design process is even more important in this context since several heterogeneous expertise domains have to work together and exchange data, take decisions based on these data...

According to [18], three ways can be used to perform this semantic interoperability: by mapping, through a mediator and by request exchanges.

The first solution consists in describing correspondences between linked semantic concepts of two different models. Since this method is currently the most used, several technical solutions are available in the literature. They are summarized in [19].

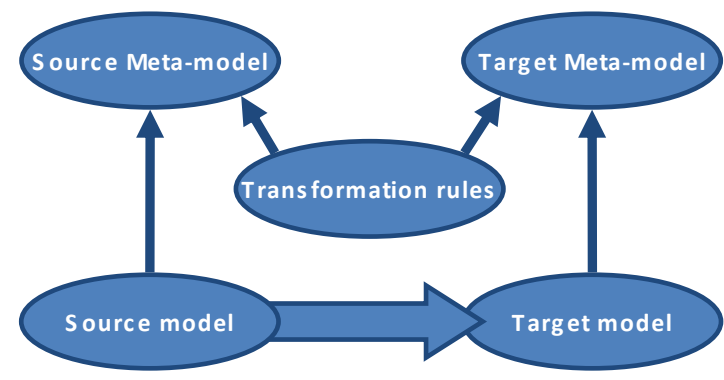

Figure 4: Meta-model transformation [20]

Adapted to models transformation, the Figure 4 illustrates that transforming source model into target model needs to specify transformation rules applied on both source and target meta-models. Obviously, an explicit representation of mapped data can help to realize this kind of correspondence.

In this context of collaborations between several heterogeneous experts and tools, several semantic conflicts can appear, such as synonymy and homonymy issues. In order to solve these semantic misunderstandings, the second solution consists in using intermediate mechanisms (as mediator, ontology or agents [21]) as references, needed to associate these different interpretations of the same concept. The idea of mediator which was firstly developed in the legal domain to solve conflicts between citizens is more particularly described in [22] and [23]. These mediators can be enriched by specific knowledge, rules and mapping schemas to coordinate information sources.

Nowadays, this mediator role is mainly performed by ontologies. Defined as an "explicit specification of a conceptualization" [24], an ontology proposes a framework to describe and formalize knowledge in order to be handled and processed by computer systems. Consequently ontology is used as a referent knowledge model independent from schemas handled by consumers, sharing vocabulary and standard communication protocols.

The third and last solution is the requests exchanges approach, based on interoperable language such as the ones derived from 
SQL and XML. Thanks to these languages, databases can be directly requested and can exchange data. This solution becomes really efficient if the semantic structure of each model requested is respected.

These three semantic interoperability approaches are currently developed and used by both industrials and research community (especially the European NoF INTEROP Virtual Laboratory). Nevertheless, each of them needs to identify, explicit and formalize all models (of each domains and tools) involved in the collaborative design process. Although this paper is not dedicated to the modeling issue and methods, it describes, in further sections, how an adaptation of Business Process Management (BPM) can be relevant to help experts to describe their processes. The next section deals with the last interoperability layer: the technical one, by summarizing existing solutions and their limits.

\section{B. Technical interoperability}

The last layer, the technical interoperability, consists in allowing different devices, networks, operating systems and applications to exchange data. Historically, this interoperability layer was wrongly considered -in the industry- as the most important as underlined by [25]: "Interoperability should not only be considered a property of ICT systems, but also concerns the business processes and the business context of an enterprise". To perform this interoperability two kinds of approaches can be used.

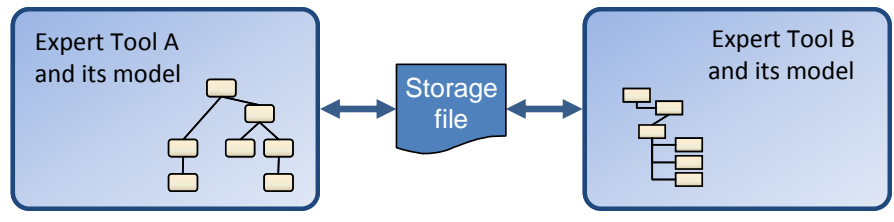

Figure 5 : Asynchronous solution

As illustrated in Figure 5, the first is based on an asynchronous file exchanges: in this case, shared data is contained in a file with a well known format which can be proprietary, neutral or open. STEP, designed by the ISO consortium (ISO 10303), mainly used to exchange geometrical data between CAD systems and XML, from the W3C consortium [26] firstly designed for data exchanges between heterogeneous databases, are two examples of neutral formats. This solution implies to develop converters to map file from the source application to the target one. The use of neutral format reduces the number of convector to develop but those formats are complex to implement. This solution is mainly used for platform to platform synchronization because this operation is a long and complex process that can be hardly done in real time.

The second approach for interoperability consists in data direct sharing and handling through a network. Compare to the first solution, exchange are synchronous. The number of solutions available is growing continuously, more since the first XML developments. Service Oriented Architecture, Web Services and the Enterprise Service Bus technology (with improve the Web service concept by adding network management) are good example of these synchronous solutions.

Web Services, which are nowadays commonly used [27], are defined as "software component encapsulating specific functionalities available through thanks to Web standard and open protocols" [26]. Since it is based on opened and well known standards like XML, Web Services ease sharing of both data and functions between very heterogeneous peers (operating system, programming framework, programming language). Exchanges between the web service consumer (which can be a website or in the study case of this paper an expert application) and its provider transit through http protocol with XML formatted messages. As illustrated in Figure 6, in order to ease the finding of the fittest service, consumers can interrogate an intermediate directory (called UDDI) which redirects their request to the provider. During the first contact, the Web service provider sends to its consumer the list of services available and the structure of handled data, formatted into a WSDL contract (expressed in XML). Then, the following exchanges can be easily performed since both consumer and provider agree on the format of data sent and received. 


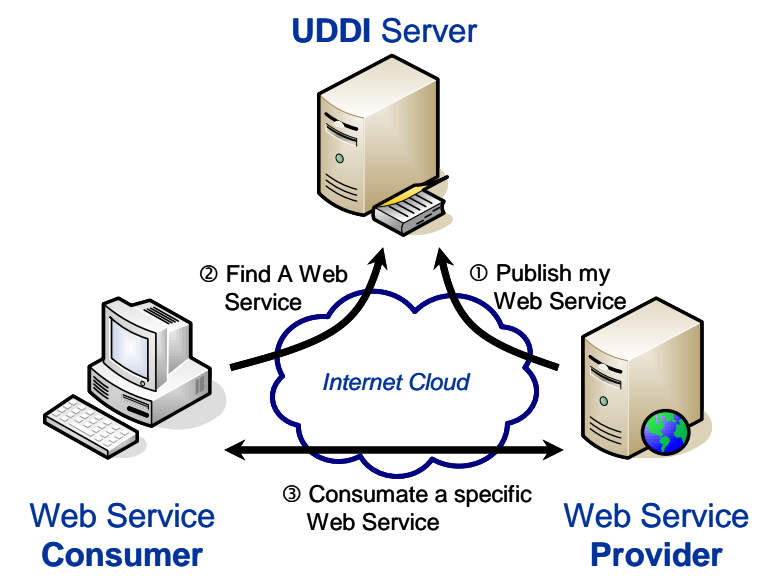

Figure 6: Web Service: the 3 main actors and theirs collaborations [26]

In this context of collaborative product design, where experts have to share and request instantaneously information into a heterogonous context, this solution of synchronous exchanges based on Web Services looks very interesting. That is the reason why the following section deals with the proposed concept called Inter-OPP (Organization Product and Process), underlining the benefits (considering semantics and technical interoperability) of opening the PPO kernel thanks to the Web Service technology.

\section{INTER-OPP: OUR DEVELOPMENT}

Dedicated to the context described in the introduction of this paper, experts developing methods, framework and tools to improve collaborative product design are mainly faced with interoperability issues. As explained previously, among the three levels of interoperability, this paper focuses on the specification of a solution easing the semantic and technical interoperability between each expert and their specific tools.

Considering the semantic interoperability, among the solutions described in previous chapters, the mediator approach seems to be relevant in this context where flexibility is needed during the whole design phases (intervention of experts).

The section II reminds that PPO (Product Process Organization) model is the result of a model synthesis of what representative French experts in product and manufacturing design handled or needed in their expertise. That is the reason why this model can be considered as an understandable and usable common model for experts of the whole product life cycle. Moreover, this section underlines that the developed PPO kernel is flexible enough (especially in product modeling) to fit specific needs expressed previously. Thanks to this flexibility, the PPO model remains the best mediator solution for semantic interoperability in product design context. The next of this chapter deals with how to improve and instrument PPO model to perform this mediation work. In addition, technical aspects of this interoperability are detailed more precisely by focusing on the dictionary and subscription concepts.

\section{A. Use Cases}

To be considered as totally interoperable in the concurrent product design context, the PPO core must succeed in four exchange scenarios. These use cases are illustrated in Figure 7:

- In Use Case 1, PPO kernel must be able to expose data (i.e. attributes of PPO objects, as for instance: dimensions, materials, physician principles, equations and so on...) to expert tools. This is the easiest step.

- In the second use case, PPO kernel must be able to integrate modifications or additions of data or whole structure from an expert tool.

- The PPO core can store files by linking them; they are considered as special attributes. For instances, a CAD Part File is an attribute of a component class in the product model. That is the reason why the third use case concerns file sharing ability. This use case is an extension of the first and second Use Case.

- Since a major objective of this project is to open interoperability to PDM systems, PPO kernel must allow them to manage access rights of files stored in the PPO core, and to add new ones. This is the last use case.

When these four steps are successfully done and so, when PPO kernel can be considered as totally interoperable, several expert tools can conceptually be indirectly interconnect through the PPO core:

- Exchanges between several expert tools,

- Exchanges between expert tools and PDM systems,

- Exchanges between PDM systems. 


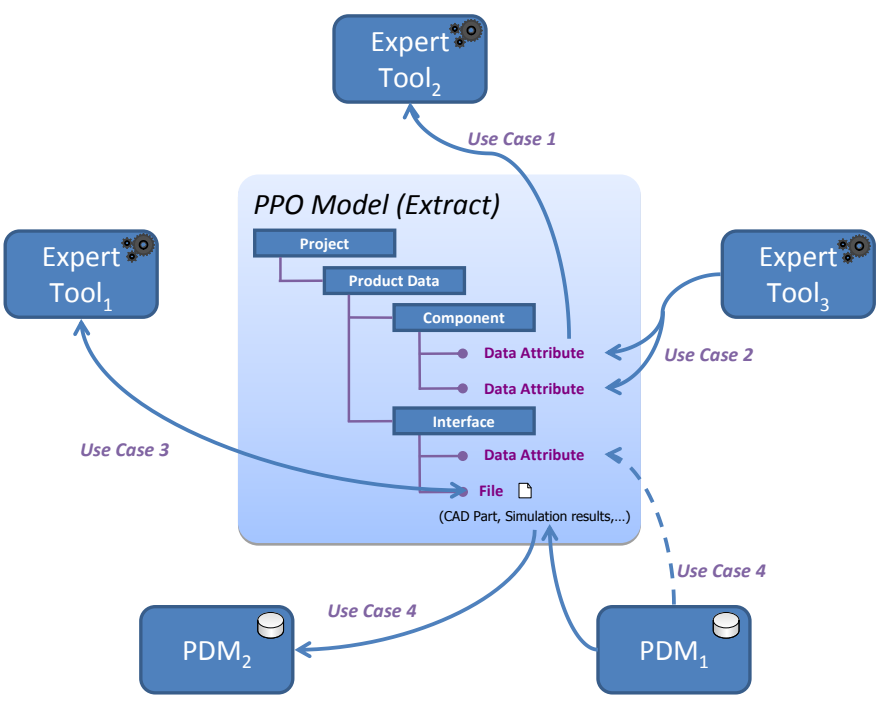

Figure 7: The four steps (noted Use Case) to interoperability between Expert Tools and/or PDM systems, based on PPO framework.

However, at this stage, it is impossible to directly connect these applications to the PPO kernel since there is no match between the expert model and the PPO model. The further sections focus on two major concepts performing this semantic and technical interoperability: dictionary and subscription concepts.

\section{B. Tools for interoperability: the dictionary and subscription concepts}

In order to describe the solution developed to realize this semantic and technical interoperability (factorized into four use cases), the Figure 8 summarizes concepts and tools chronologically needed in the Use Cases expressed in the previous section. Moreover, symbols are added to show the job status for each stage. As illustrated, four main steps are needed to perform exchanges between expert tools and the PPO kernel:

(1) Modeling and structuring information handled by experts and their tools they want to connect to PPO kernel. This stage can be performed, for instance, with UML language or a simple XML file. This modeling process could be assimilated to the MDA (Model Driven Architecture) paradigm [28] or the Model Driven Engineering (MDE).

(2) When the whole model is performed, experts have to select data to share. Even if this selection is technically easy (extraction of data contained into a database or formatted files such as XML ones), the real difficulty is to identify what are those relevant data. Some methods of the BPM community [29] can be used to detect data exchanges between activities.

(3) The third stage consists in performing the semantic mapping between the expert selected and formatted knowledge and the PPO kernel. This semantic transformation stage is the major issue.

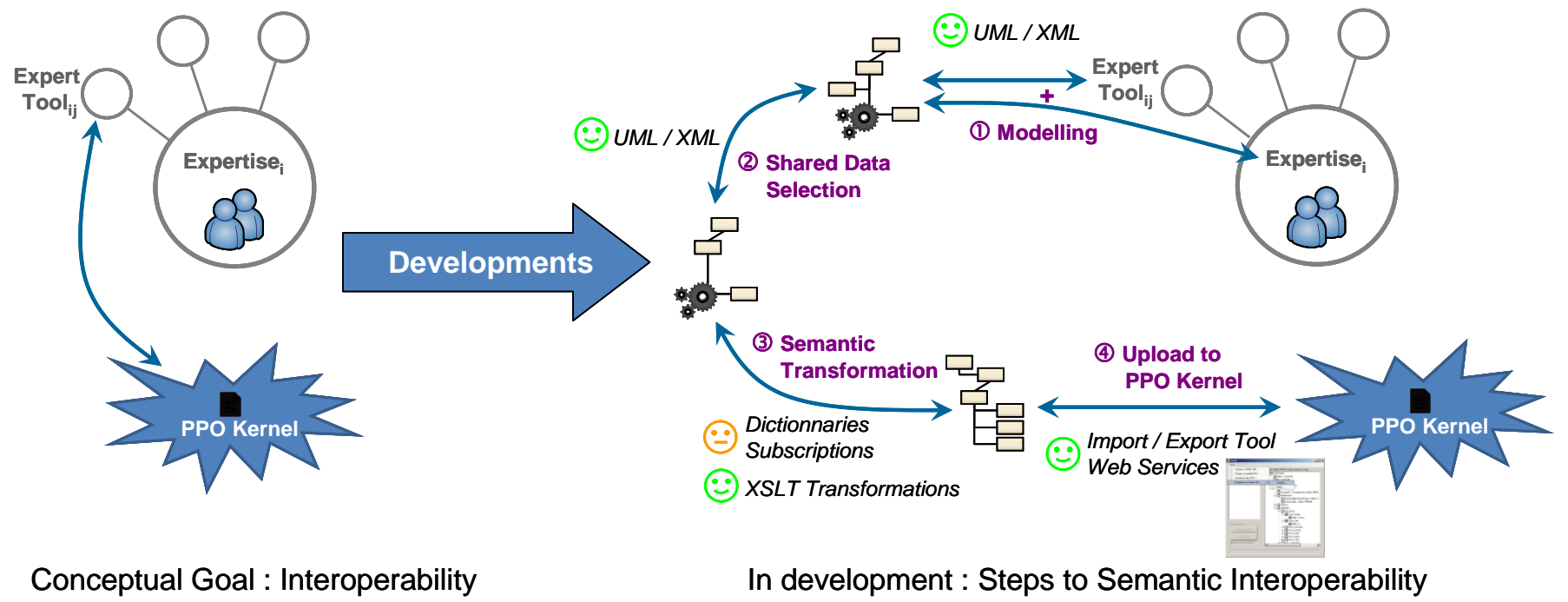

Figure 8: Comparison between conceptual interoperability and its software development

Two concepts (called dictionary and subscription

tool) supported by several technical solutions (such as 
XSLT language) are proposed to carry out this step.

(4) The last step consists in transferring the PPO understandable knowledge to the PPO kernel. Several dedicated functions based on the PPO framework detailed in section II coupled with Web Services can technically perform this stage.

As explained at point (3), models transformation (from expert to PPO) is based on two major concepts: the use of dictionary to extend the scope of PPO model and the subscription tool that aim to simplify the mapping between PPO and expert applications. The next sections describe more particularly each of them.

\section{1) Dictionary files}

As explained in section II.A, components of the PPO product model are simple enough to fit with information handled by any expert tool. However since each expert handles specific information and knowledge PPO product model must be extended to integrate these structures.

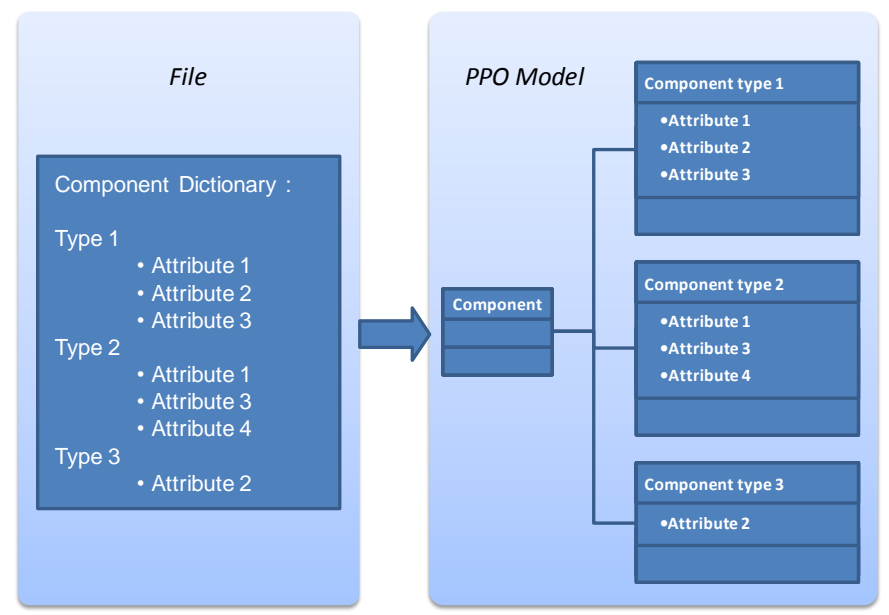

Figure 9: Extend IPPOP model with dictionary file

To carry out such adaptation, the PPO framework allows defining dictionary files. Thanks to these files, experts can customize each component of PPO model in order to fit it.

As illustrated in Figure 9, the dictionary is just a text file which specifies several component types and their attributes. PPO is then able to read this file and to add the instantiations of each component type into its model.

This functionality eases the addition of new expert applications thanks to this dynamic PPO extension. Nevertheless, the identification of what have to be exchanged between experts and tools supporting this operation must be developed. In order to ease the definition of the correspondences between expert tools and PPO kernel, a subscription tool is proposed and described in the next section.

\section{2) Subscription tool}

As described in III.A, interoperability between expert tools (and so by extension between these tools and PPO kernel) requires writing transformation rules. Generally, this mapping is statically contained into a converter device (an example this kind of converter is detailed in [30]) which is coded by a computer science expert who usually does not master the knowledge he converts. Since this project aims to be used by people which are not expert in data conversion, this constraint implies to develop a method and a tool which ease this mapping, and thus, the expert tool connection to the PPO mediation. This solution is called the "Subscription Tool".

In the same way that in web service technology, the WSDL contract is sent at the first connection of the client to the service provider, the use of this tool is only needed at the first connection of the expert tool. The aim of this subscription tool is to provide a human machine interface for the mapping between PPO and expert software: thus product design experts can choose, without particular IT skills, the information from PPO they want to download to their expert tools.

Although it has been conceptually validated on several industrial cases, this subscription tool is still under development. As illustrated in Figure 10, the subscription tool displays all subscriptions already done by experts in the subscription list. For each of them, expert can precise for each class of the knowledge needed by his expertise and tool the PPO class to associate. Thanks to this subscription interface, mapping on attributes (characteristics), structures and objects can be defined. If the user does not find the component he needs in PPO kernel, he can create an extension of PPO model thanks to the dictionary concept described in the previous section.

When this association is performed, the subscription tool generates a file required for the mapping procedure and writes it on PPO server. The structure of expert model is then written on the server. From now, for each connection on PPO kernel, the dedicated information, structured as expressed by experts can be exchanged: expert application has just to connect to PPO server, give its subscription number and receive the subscribed data

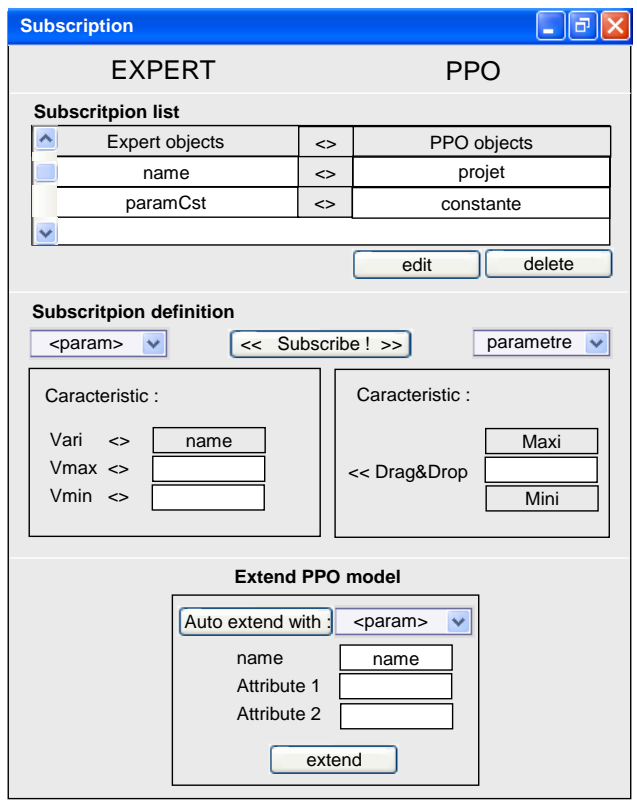

Figure 10: Draft of subscription tool user interface.

A strong need of this solution is that expert applications can 
export and import data in XML format. This need is not industrially incredible since more and more software can easily manage XML format. In addition, several software frameworks such as BizTalk [31] provide tools to model, manage and convert data (contained in XML file) between mainly databases. Moreover this constraint is a good way to assure that experts, and so the final users of the PPO kernel, express rationally their needs (expressed in XML format or thanks to DTD format).

As a conclusion, the Figure 8 underlines perfectly the gap between the interoperability needs and its software developments. Although this paper suggests two concepts to ease semantic interoperability: the dictionary and subscription concepts, these software developments are still uncompleted and need to be validated with several industrial cases fitting the use case summarized in the Figure 7. That is the aim of the next part: three cases are proposed to illustrate how this InterOPP approach is interesting in a product design framework.

\section{ILLUSTRATIVE CASES}

In order to validate conceptual and technical choices, several applications of this interoperability approach by mediator were carried out to industrial and research issues. Three of them are more detailed in the further sections: each of them illustrate one use case expressed previously in Figure 7.

\section{A. Robustness Analysis Tool}

The first step to the development of the whole Inter-OPP approach consists in proving the feasibility of the first use case (expressed in Figure 7): PPO model exposure and its manipulation. To validate this scenario, the PPO kernel is plugged to a robustness analyser tool developed by the French ODIC laboratory and Delta-CAD company. The aim of this plug is to provide, in the context of collaborative design of switch MEMS [32], to this expert tool a mean to access and use data from the product model contained into the PPO kernel.

Thanks to the IPPOP platform and its web interface (as illustrated in Figure 3) this scenario can already be played. Indeed through web user interface, expert can interact with information contained into PPO kernel: so he can manually consult these data, extract them into its robustness application and evaluate the design solution relevance. Then, if needed, he can enrich the product model with its results by manually putting them into the PPO kernel. This scenario is noted 1 in Figure 11.

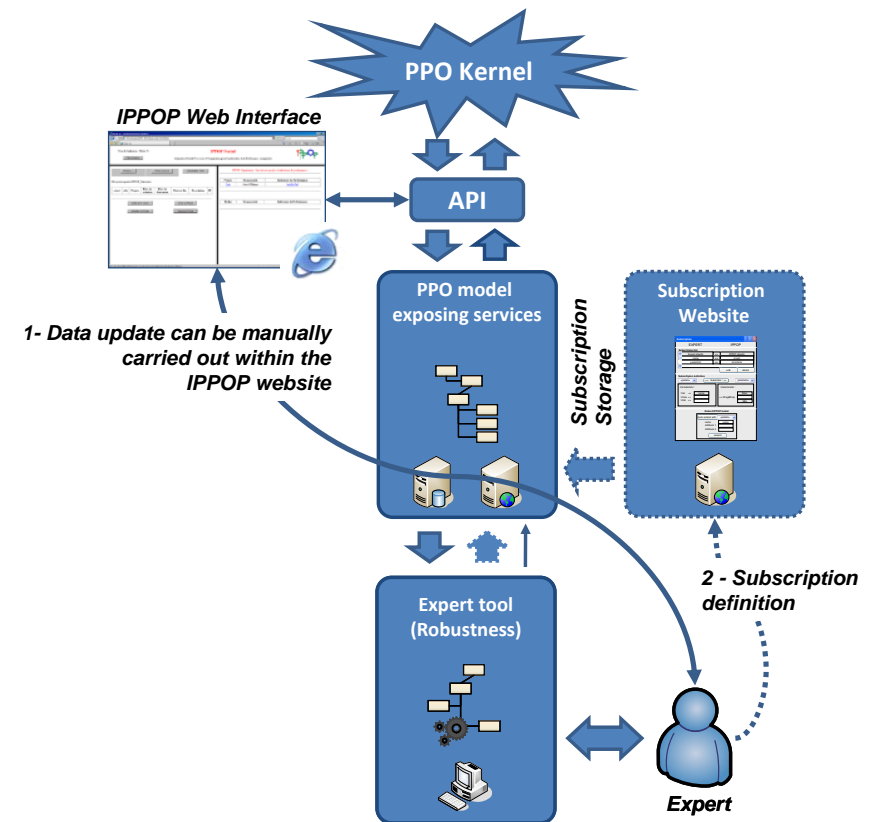

Figure 11: Interoperability architecture and exchanges between the robustness analysis tool and the PPO kernel

Moreover this figure illustrates, in the framework detailed in the IV.B, what have been developed. Doted border squares correspond to solutions conceptually validated but not implemented or integrated to the Inter-OPP framework for this research case (especially the automated and distant subscription tool). Chronologically, the information needed to define further exchanges is done in 3 stages:

- To perform their analysis, robustness experts handle three kinds of object: influent design parameters associated with their variability attributes, performance parameters which model the robustness analysis criteria and the mathematical function linking all of these parameters. The first step consists in modeling and structuring these concepts. Then this model is expressed by using the subscription user interface given in Figure 10 to associate these objects with the ones contained into the PPO kernel or into dictionaries.

- When this association is completed, the subscription is stored in the PPO server; its ID number is generated and given to the robustness expert: no further settings are needed.

- At this stage, the PPO server can send robustness parameters in the format specified by the user as soon as its ID is called by any distant application. This data exchange is performed thanks to a combination of web services. In order to be compliant with the most software solutions, two ways are available to get information: the handling of formatted objects sent by the web service or the download of a XML file. Both meet the format expressed by experts in the first step. The Figure 12 illustrates the result of a XML file download from the PPO kernel, considering the subscription associated with the robustness analysis tool. 


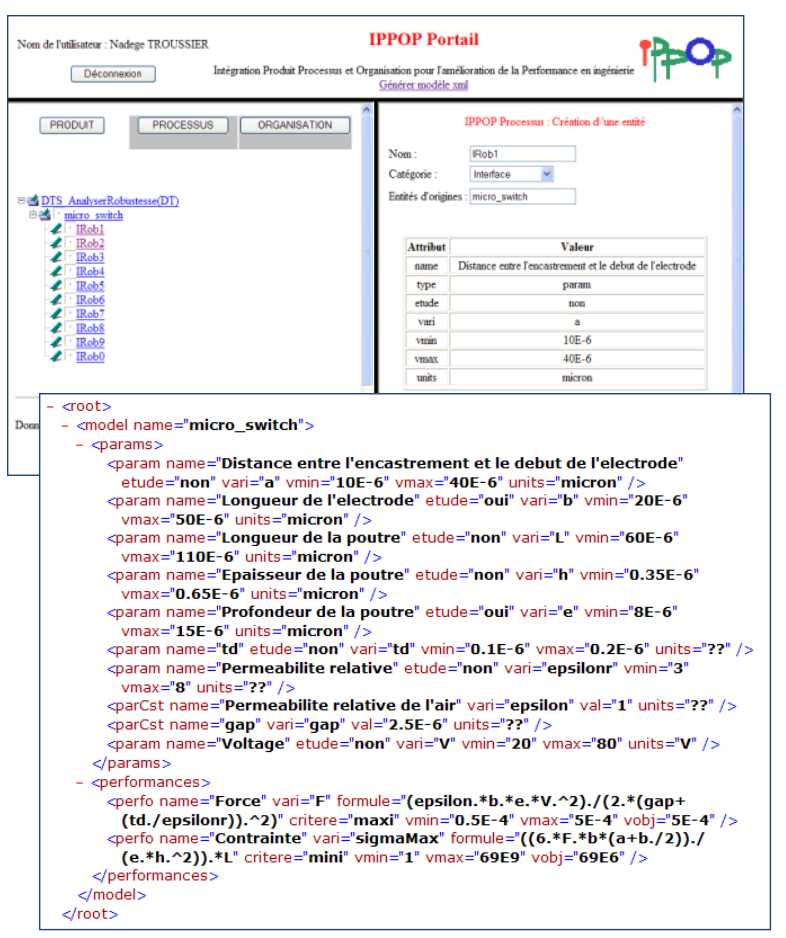

Figure 12: Screenshots of information contained in the PPO kernel (displayed with the web user interface) and its extraction fitting the robustness analyzer requirements

Although the whole process is not completely integrated, each step has been validated thanks to several software developments. The main scenario work fine and several demonstrations of loading into the robustness tool information previously modified by using web user interface were performed. However, the first step of modeling expert processes is not yet automated: the mapping between PPO concepts and robustness one is not the result of the expression of robustness experts through the subscription tool but still the implementation of mapping rules defined and coded by a computer science expert.

As a conclusion, even if it was not totally integrated, this first implementation on robustness expertise (domain totally unknown by the designers of Inter-OPP) validates concepts and method animating this interoperability through mediator approach. The next section deals with the second application of this approach to Computer Aided design tools which are mainly used in product collaborative design.

\section{B. Computer Aided Design}

In order to validate the ability of PPO kernel to expose and modify stored information (that is the first and second use cases defined in Figure 7), another experiment was performed in the framework of Computer Aided Design by Troyes University students. The scenario followed consists in updating Product parameters manipulated by a CAD system (in this case CATIA) thanks to information exposed by PPO kernel.

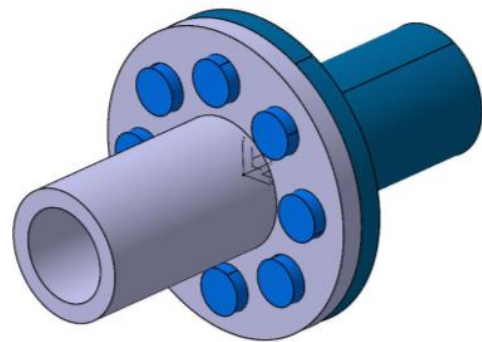

Figure 13 : The coupling assembly

The mechanical test part, which belongs to a part family, is a simple screwed coupling assembly composed of two main shafts and several screws. An illustration is given in Figure 13. Consequently, a workpiece belonging to this family can be described with six design parameters available in CATIA (as, for instance: screws diameter and number, and so on...).

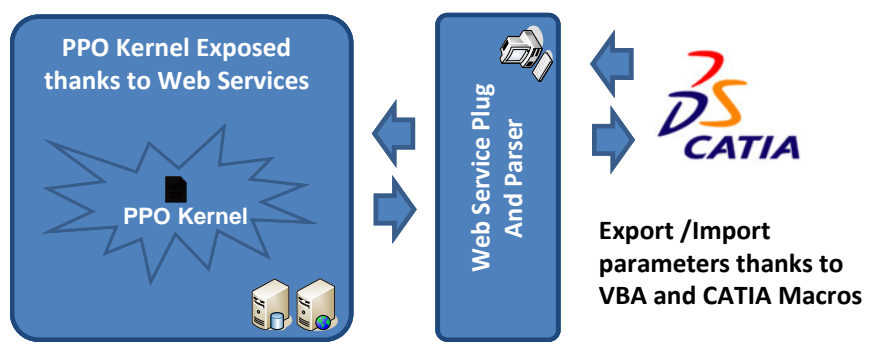

Figure 14: Import/Export Product parameters scenario between CATIA and PPO kernel

This dedicated scenario (illustrated in Figure 14) follows the same way than the solution used to perform the robustness analysis tool which is explained in section V.A. The only technical difference is due to the difficulty to directly implement consumption of Web Services with the programming language available in the CATIA framework (which is an extension of VBA functions). Consequently, a smart plug is needed and placed between the PPO information provider and CATIA consumer.

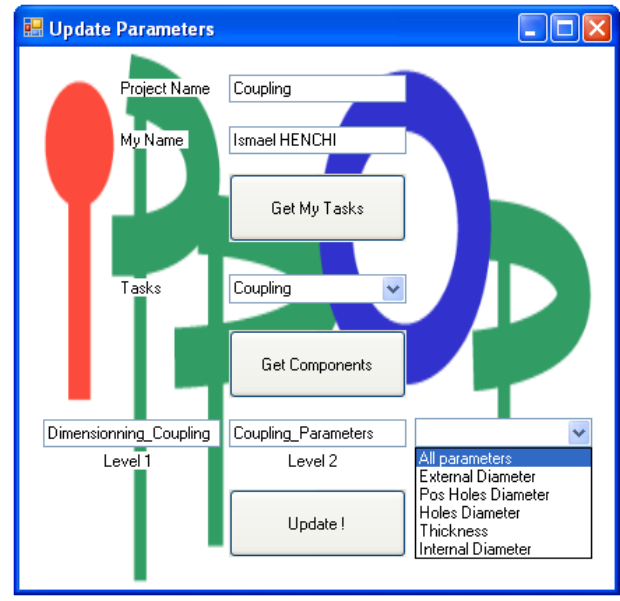

Figure 15 : User Interface displayed in the CAD system

As a result, designer can easily update selected parameters (or all if he wants) by using interface as illustrated in Figure 15: the update process is totally transparent, since mapping rules are dynamically generated from models included into the subscription tool. 
Nevertheless this subscription model is still directly coded into this added plug and is not yet the result of the identification and capitalization of the final user (in this case: designers) needs. The aim of this subscription tool is to provide to designers the easiest way to match their own design parameters with information provided by the previous design phases (emerging from the functional requirements or the customer needs for instance), which are gathered into the PPO kernel. Several improvements and developments are needed to completely validate this process and solutions.

\section{PDM to PPO interoperability}

The third validation takes place in the framework of the French project called SEINE [33] (meaning "Standard for Innovative Digital Extended Company"). Among the several goals of this project, it aims to standardize and improve exchanges (considering both data and process views) between Product Lifecycle Management systems in order to ease collaborative engineering between OEM and suppliers in the aeronautics framework.

This project leads to the definition of standards, models and processes needed to manage high level exchanges, mainly the Product structure. Nevertheless, lower level collaboration (handling files, Product data and parameters) is not taken into consideration at this stage of the project. Considering the PPO model validity domain, it remains a good way to ease these lower level collaborations: that is why several works were ignited to plug PLM systems to PPO kernel [34]. These developments implement the fourth use case given in Figure 7.

Concepts, solutions and works performed in this project are summarized in [35]. To validate this interoperability between PLM systems and the PPO kernel, works focused on a simple scenario: generate the product structure in a commercial PLM system, WindChill, directly from the product structure stored and shared by the PPO model. This PLM system was selected due to its ability to consume Web Services.

For this validation case, the modelling work leads to the definition of transformation rules between the PPO model and the WindChill project model. These rules, which should be automatically generated by the subscription tool, are still defined manually. Several rule examples are given in Figure 16.

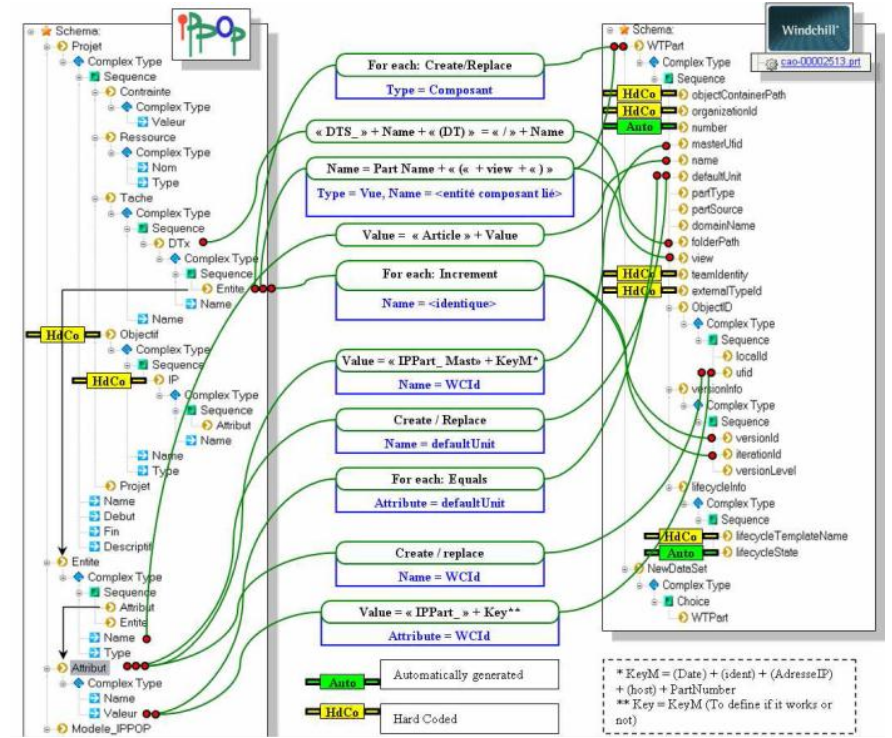

Figure 16 : Correspondence description between IPPOP and WindChill structures.

This mapping, which usually belongs to the technical interoperability, is faced with semantic issues since the concept of Product and tasks are not the same in WindChill and PPO: there are not considered in the same level! This correspondence work is thus the key activity in the Inter-OPP process: completing the subscription tool is the real challenge!

This use case was technically implemented by using Web Services exposed by the PPO kernel. Since the mapping could not be performed by using subscription concept, this process was based on a powerful and free XML transformation language: XSLT [36]. The use of this kind of language truly complies with the web services since they both handle XML in order to structure data and information.

To validate this link between PPO (handling mainly Product data and information) and WindChill (managing the project and company organisation), an application case was defined: the portative drill machine (originally proposed by [4] and [6]). The result of exchanges between these two systems is given in Figure 17. This shows that WindChill and PPO information trees remain totally different: the WindChill EBOM tree does not take into account information concerning functions or component interfaces... Moreover, its underlines the proper data granularity of each system: where PPO kernel stores and exposes both product parameters and their containers (mainly files), WindChill only manages files and their metadata. 


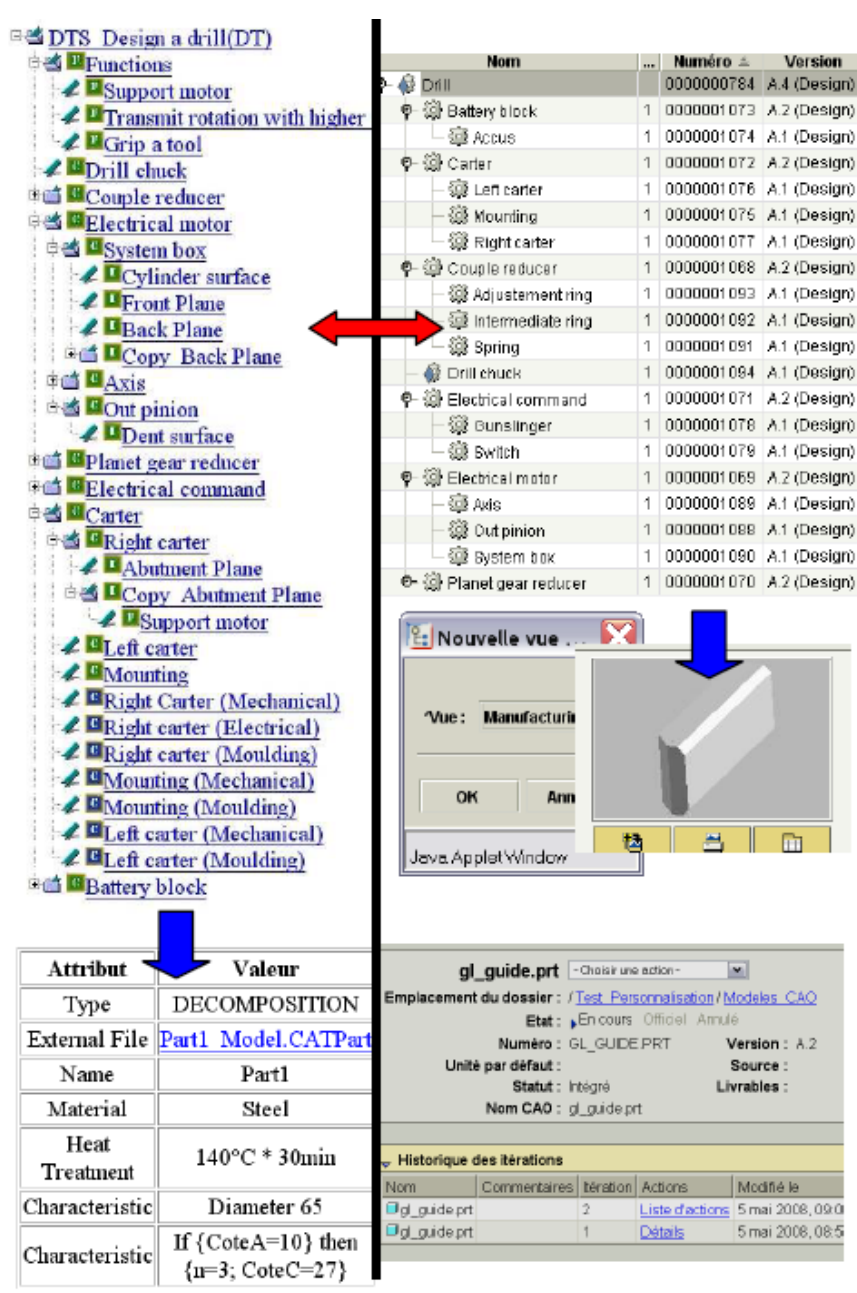

Figure 17: Exchanges and managed information

These application cases validate several concepts and technical solutions of Inter-OPP. Moreover it emphasizes that this idea is both technically and conceptually interesting since it allows to several dedicated tools (and very often limited to their expertise domain) to exchanges data and information through the PPO mediator. Nevertheless, futures works should mainly focus on the subscription tool with remains the key concept of Inter-OPP.

\section{CONCLUSION}

As a conclusion, this paper deals with an interoperability framework for collaborative design between distant and heterogeneous expert tools. Based on the PPO kernel developed by the French national IPPOP Project, which is flexible enough to be used by most of the expert applications, this solution proposes to map expert models thanks to a user friendly interface. The dictionary concept, which allows creating expertise dedicated views from the elementary concepts contained in the PPO kernel and the subscription tool ease the mapping process, usually synonymous of interoperability.

A first PPO server demonstrator has been deployed and enriched with web services technology: several use cases were validated thanks to their application on industrial and research cases such as robustness analysis or PDM interoperability. These demonstrators which remain the first step to the validation of the whole approach underline several limitations and issues we have to overcome.

Thus, a huge conceptual issue must be solved: the too permissive freedom allowed by the PPO model. Indeed, due to its flexibility, experts from heterogeneous domains must work together and agree on the overall product structure to be adopted: parameters linked to assembly, those linked to parts... This issue is more crucial in this inter-OPP approach where experts themselves generate the mapping rules between their expertise and the common product model through the subscription tool! Several solutions can rise by drawing on concepts developed in the MDA (Model Driven Architecture) community.

In addition, technical improvements have to be continued: especially the design of the user interface of the subscription tool. These developments must be performed since the semantic interoperability is partially based on this concept!

Moreover the decisional dimension is not yet taken into account by this Inter-OPP framework. Nevertheless, this activity could be considered as one another expertise that has to be plug to the PPO kernel. Indeed this activity needs indicators displaying that the situation can be considered as good as estimated by accessing selected and strategic data contained into the PPO kernel. Only the orchestral dimension (how to allocate and to sequence interventions of the different expertise) is not really regarded and must be a further way to explore.

Parallel to the development of the PPO server, the second objective is to keep federating experts and knowledge (in the same way than the national French project IPPOP) in order to develop and consolidate an open source platform, based on the PPO kernel. This community of users, which wants to raise this platform as a reference in the computer aided engineering domain, is called PICS-PPO (a French acronym for: Integration Platform for the Knowledge Management and the Simulation of the Product-Process-Organization Behavior in Engineering).

\section{ACKNOWLEDGMENT}

We sincerely thank members of the ODIC laboratory from Compiegne Technological University for our enriching exchanges about the robustness analysis.

\section{REFERENCES}

[1] G. Sohlenius, Concurrent Engineering, Annals of CIRP, Vol 41(2), 1992, pp. 645-655.

[2] G. Ducellier, S. Charles, B. Eynard, E. Caillaud, Traceability of simulation data in a PLM Environment: proposition of Step-based system that support parameter integration, in Proceeding of International Design Conference - DESIGN 2006, Dubrovnik, Croatia, 2006, p511-518. 
[3] IPPOP Project WebSite, http://ippop.laps.u-bordeaux1.fr/, last visit 05/27/09.

[4] F. Noël, L. Roucoules and D. Teissandier, Specification of product modeling concepts dedicated to information sharing in a collaborative design context, in Proceeding of IDMME 2004, Bath, UK, 2004, pp. 112.

[5] L. Roucoules, F. Noël, D. Teissandier, M. Lombard, G. Débarbouillé, P. Girard, C. Merlo and B. Eynard, IPPOP: an open source collaborative design platform to link product, design process and industrial organization information, in Proceeding IDMME 2006, Grenoble, France, 2006.

[6] J. Dufaure and D. Teissandier, Integration of a tolerance analysis tool (MECAmaster) in a Tolerancing framework based on IPPOP, an open collaborative platform, in Proceeding of IDMME 2006, Grenoble, France, 2006.

[7] B. Rose, Proposition d'un référentiel support à la conception collaborative: $\mathrm{CO}^{2} \mathrm{MED}$ (Collaborative Conflict Management in Engineering Design), Prototype logiciel dans le cadre du projet $I P P O P, \mathrm{PhD}$ thesis of the Henri Poincare University, Nancy, France, 2005.

[8] ISO/IEC 23821, Information technology Vocabulary. Part 1: Fundamental terms. ISO/IEC Standard, Geneva, Switzerland, 1993.

[9] IEEE, Standard computer dictionary, A compilation of the IEEE standard computer glossary, IEEE Standards, New York, ISBN: 1559370793, 1990, pp. 610.

[10] IEEE - Standards Information Network. IEEE 100, the Authoritative Dictionary of IEEE Standards Terms, Seventh Edition, New York, 2000.

[11] F. Vernadat, Enterprise Modeling and Integration: principles and applications, Chapman \& Hall, ISBN: 041260550 3, 1996.

[12] European eGovernment Services, Interoperable Delivery of European eGovernment Services to public Administrations, Businesses and Citizens (IDABC), November, Luxembourg (Website: http://ec.europa.eu/idabc/).

[13] D. Konstantas, J.-P. Bourrières, M. Léonard, N. Boudjlida, Preface of Interoperability of Enterprise Software and Applications, Proceeding of INTEROPESA'05, Geneva, Switzerland, Springer-Verlag, p. 5-6, 21-25 February 2005.

[14] ATHENA: Advanced Technologies for Interoperability of Heterogeneous Enterprise Networks and their Applications, Interoperability of Enterprise Software and Applications, Springer, London, ISBN: 978184628151 8, July 2006.

[15] IDEAS: Interoperability Development for Enterprise Applications and Software, A gap Analysis -Required activities in Research, Technology and standardization to close the RTS Gap-Roadmaps and Recommendations on RTS activities, IDEAS, available on http://www.ist-world.org/, 2003.

[16] European Interoperability Framework, White Paper, Brussels, http://www.comptia.org, February 2004.

[17] S. Pokraev, D. Quartel, M. W.A. Steel, M. Reichert, Semantic Service Modeling: Enabling System Interoperability, Enterprise Interoperability: New Challenges and Approaches, Springer Verlag, ISBN: 1846287138, 2006.

[18] J. Park and S. Ram, Information System Interoperability: What lies beneath?, ACM Transaction on Information System, Vol. 22(4), 2004.

[19] E. Rahm and P.A. Bernstein, A survey of approaches to automatic schema matching. The VLDB Journal, Volume 10(4), 2001, pp. 334350.

[20] R. Lemerles, Transformation Rules based on Meta-modeling, IEEE Enterprise Distributed Object Computing Conference (EDOC), La Jolla, California, 1996, p113-122.

[21] S. Papastavrou, E. Pitoura and G. Samaras, Mobile agents for $W W W$ distributed database access, in proceedings of the $15^{\text {th }}$ International conference on data engineering, Sydney, Australia, March 1999.

[22] B. Bauer, J.P. Müller, S. Roser, Adaptive design of cross organizational business processes using a model-driven architecture, International Conference on Business Information Systems, Physica-Verlag, 2005, pp. 103-121.

[23] J.L. Lascoux, Pratique de la médiation, ESF, Nanterre, France, ISBN: $2710118599,2007$.
[24] T.R. Gruber, Toward principles for the design of ontologies used for knowledge sharing, In Formal ontology in conceptual analysis and knowledge representation, Kluwer academic publishers, 1993.

[25] A-J. Berre, B. Elveaster, N. Figay, C. Guglielmina, S. G. Johnsen, D. Karlsen, T. Knothe and S. Lippe, The ATHENA Interoperability Framework, Enterprise interoperability: New challenges and approaches II, Springer edition, ISBN: 978-1-84628-857-9, 2007.

[26] W3C (World Wide Web Consortium) recommendations on Web Service, dedicated Website: http://www.w3.org/2002/ws/, last consultation on September 2009.

[27] J. L Zhao, H. K. Cheng, Web services and process management: a union of convenience or a new area of research?, Decision Support Systems, Volume 40, Issue 1, July 2005.

[28] R. CHOLEY and the OMG (Object Management Group) Staff Strategy Group, Model Driven Architecture White Paper, available on the OMG Website: http://omg.org/, November 2000.

[29] BPMI, Business Process Modelling Notation (BPMN), Version 1.0, available on the BPMI Website: http://www.bpmi.org/,May 3, 2004

[30] H. Song, L. Roucoules, B. Eynard and P. Lafon, Interoperability between cooperative design modeler and a CAD system: software integration versus data exchanges, International Journal for Manufacturing Science and Production, Vol. 7(2), 2006.

[31] Microsoft BizTalk 2009 White Papers and technical information, http://www.microsoft.com/biztalk/en/us/white-papers.aspx, last consultation on October 2009.

[32] J.S. Klein Meyer, G. Cabannes, P. Lafon, N. Troussier, L. Roucoules, T. Gidel, Product modeling for design alternatives selection using optimization and robustness analysis, International Conference on Engineering Design (ICED’07), august 2007, Paris, France.

[33] SEINE, website describing the project, actors, deadlines and objectives, http://www.telecom.gouv.fr/fonds_documentaire/ticpme2010/seine.pdf, displayed in October 2009.

[34] D. Van Wijk, B. Eynard, N. Troussier, F. Belkadi, L.Roucoules, G. Ducellier, Integrated Design and PLM Applications in Aeronautics Product Development, Proceeding of the CIRP Competitive Design Conference, Cranfield University, March 2009.

[35] D. Van Wijk, A. Etienne, E. Guyot, L. Roucoules, B. Eynard, Enabled virtual and collaborative engineering coupling PLM system to a product data kernel, Proceeding of the $5^{\text {th }}$ International Conference on Digital Enterprise Technology 2008, Nantes, France, 22-24 October 2008.

[36] W3C (World Wide Web Consortium) recommendation on the XSL Transformations (XSLT) v2.0, 23 January 2007, http://www.w3.org/TR/xslt20/, last consultation October 2009. 